\title{
Kruppel-Like Factor 2 Regulates Dendritic Cell Activation in Patients with Acute Coronary Syndrome
}

\author{
Hongcheng Fang ${ }^{\mathrm{a}}$ Jing Lin ${ }^{\mathrm{b}, \mathrm{c}}$ Lei Wang ${ }^{\mathrm{a}}$ Peiyi Xie $^{\mathrm{a}}$ Xiaoyan Wang ${ }^{\mathrm{a}}$ Jianyun Fu ${ }^{\mathrm{a}}$ \\ Wen $\mathrm{Ai}^{\mathrm{a}}$ Shaoyuan Chen ${ }^{\mathrm{a}}$ Fei Chen ${ }^{\mathrm{a}}$ Fengxiao Zhang ${ }^{c}$ Yousu Su ${ }^{\mathrm{a}}$ Dazhu $\mathrm{Li}^{\mathrm{c}}$
}

\begin{abstract}
aDepartment of Cardiology, Shenzhen Sixth People's Hospital (Nanshan Hospital), Huazhong University of Science and Technology, Union Shenzhen Hospital, Shenzhen, bDepartment of Cardiovascular Medicine, Shaanxi Provincial People's Hospital, Xi'an, 'Department of Cardiology, Institute of C Diseases Cardiovascular, Union Hospital, Tongji Medical College, Huazhong University of Science \& Technology,

Wuhan, China; The first three authors contributed equally to this work
\end{abstract}

\section{Key Words}

$\mathrm{KLF} 2 \cdot$ Atherosclerosis $・$ Dendritic cell activation $\bullet N F-K B$ pathway

\begin{abstract}
Objective: Dendritic cells (DCs) activation is important in atherosclerosis and coronary heart disease, but the mechanisms regulating activation of dendritic cells remain largely unclear. The aim of this study was to evaluate the effect of transcription factor Kruppel-like factor 2 (KLF2) in the proinflammatory activation of DCs in acute coronary syndrome. Methods and Results: In this study, the expression of CD80 and KLF2 was detected in DCs in normal health controls, patients with stable angina (SA), and acute coronary syndrome (ACS). Our study found that compared with normal control and SA, KLF2 expression in DCs is reduced in patients with ACS. Moreover, the surface expression of CD80 was increased in ACS. In vitro experiment, we found that ox-LDL could increase CD80 expression and decrease KLF2 expression. Furthermore, down-regulated KLF2 could in turn increase CD80 expression via NF-KB pathway. Conclusions: These observations identify KLF2 as a novel negative regulator of DC function and it may play an essential role in DC activation in ACS.
\end{abstract}

Copyright (C) 2013 S. Karger AG, Basel

\section{Introduction}

Atherosclerosis (AS) is a multifactorial process and one of its outcomes, coronary heart disease (CAD), is the leading cause of death worldwide. Atherosclerosis involves endothelial dysfunction, genetic and hemodynamic factors as well as other acquired and modifiable risk

Yousu Su

and Dazhu Li
Department of Cardiology, Shenzhen Sixth People's Hospital (Nanshan Hospital), Huazhong University of Science and Technology, Union Shenzhen Hospital, Shenzhen (China), E-Mail w7717@163.com; and Department of Cardiology, Institute of Cardiovascular Diseases, Union Hospital, Tongji Medical College, Huazhong University of Science and Technology, Wuhan (China), E-Mail lidazhuhp@sohu.com 
factors, including smoking, hypercholesterolaemia, diabetes mellitus and hypertension. Now, it is clear that both innate and adaptive immune systems are involved in the initiation and progression of atherogenesis [1,2]. Dendritic cells (DCs) play an important role as professional antigen-presenting cells in the immune system because of their unique ability to induce a primary immune response by activation of naive T cells [3]. DCs are positioned at the interface of innate and adaptive immune system. They are indigenous residents of healthy arteries and are typically localized in the sub-endothelial space as well as at the media-adventitia junction [4,5]. Once sufficiently activated, vascular DCs may present autoantigens to $\mathrm{T}$ cells and initiate inflammatory responses directly in the arterial wall, and thus lead to the inflammatory process of atherosclerosis [6]. We had shown in our previous study that DCs were activated in ACS via the Toll-like receptor4/MAPKsignal pathway [7]. Although there are reports of various studies about their inflammatory mechanisms in AS, DC activation still remains largely unknown.

Kruppel-like factors (KLFs) are members of the zinc finger family of transcription factors that have been implicated in the regulation of cellular growth and differentiation [8, 9]. KLF2 is an essential tonic repressor of myeloid cell activation. It is reported that KLF2mediated regulation of the NFKB-HIF- $1 \alpha$ axis controls cellular activation and is a critical determinant of the organism's response to infection and endotoxic shock [10]. KLF2 is also a key regulator of endothelial and monocyte/macrophage pro-inflammatory action. In endothelial cells, KLF2 expression is induced by laminar shear stress and inhibited by proinflammatory cytokines. Sustained over-expression of KLF2 in endothelial cells results in the induction of endothelial nitric oxide synthase (eNOS) and thrombomodulin (TM) expression, and reduces cytokine-mediated activation of proinflammatory genes such as vascular cell adhesion molecule-1 (VCAM-1) [11]. All these factors play a negative role on modulating AS. Furthermore, recent studies have indicated that KLF2 inhibits monocyte pro-inflammatory gene expression and phagocytosis [12]. On the basis of these observations, KLF2 has been proposed as a candidate of atheroprotective factor. However, the exact mechanism of KLF2 in AS, especially on DCs remains unknown.

In our present study, we investigated the expression of KLF2 and DCs activation in patients with CHD, and provide evidence to explore the negatively modulating role of KLF2 on DCs in patients with ACS.

\section{Materials and Methods}

Subjecs

This study was approved by the Ethics Committee of Tongji Medical College, Huazhong University of Science and Technology, China. All patients gave written informed consent and research was conformed to the guidelines of the declaration of Helsinki and its amendments.

A total of 60 consecutive patients who were admitted to the Cardiology Department of Union Hospital Wuhan, Hubei, PR China, from April 2012 to October 2012 for assessment of angina pectoris were recruited for the study. Of these, 30 had ACS and 30 had SA. Diagnosis of ACS and SA was performed according to established guidelines [13]. In addition, 30 healthy volunteers with atypical chest pain and normal coronary arteries on angiography were considered as controls. All participants underwent coronary angiography after admission. Cardiovascular-interrelated factors, such as age, gender, body mass index (BMI), blood pressure, heart rate (HR), and ejection fraction (EF), etc., were estimated via physical examination, electrocardiogram (ECG), and ultrasonic cardiography (UCG). Clinical data of the patients in the study are provided in Table 1.

Exclusion criteria were: no patients had HIV, advanced liver disease, chronic-immunemediated diseases, renal failure, thromboembolism and infections during last four weeks. All participators were not administrated with anti-platelet, anticoagulant and anti-inflammatory drugs therapy for seven days prior to the study. 
Table 1. Clinical characteristics of the patients. Data are presented as mean \pm SEM or a percentage. * indicated vs normal control, ${ }^{*} P<0.05$; ${ }^{* *} P<0.05$. \# indicated vs SA group; $\#: P<0.05$

\begin{tabular}{lccc}
\hline \multicolumn{1}{c}{ Characteristics } & $\begin{array}{c}\text { control } \\
(\mathrm{n}=30)\end{array}$ & $\begin{array}{c}\text { SA } \\
(\mathrm{n}=30)\end{array}$ & ACS (n=30) \\
\hline Age (years) & $49 \pm 16$ & $60 \pm 13$ & $58 \pm 11$ \\
Sex (male/female) & $20 / 10$ & $18 / 12$ & $19 / 11$ \\
Risk factors & & & \\
Hypertension, n (\%) & $9(30)$ & $18(60)$ & $14(47)$ \\
Diabetes mellitus, n (\%) & $2(7)$ & $5(17)$ & $8(27)$ \\
Smoking, n (\%) & $7(23)$ & $14(47)$ & $13(43)$ \\
Obesity, n (\%) & $5(17)$ & $7(23)$ & $8(27)$ \\
Hyperlipoproteinemia, n $\%)$ & $4(13)$ & $12(40)$ & $14(47)$ \\
Coagulant function & & $12.65 \pm 0.58$ & $12.60 \pm 0.42$ \\
PT (s) & $12.54 \pm 0.73$ & $36.45 \pm 4.27$ & $38.07 \pm 4.72$ \\
APTT (s) & $34.20 \pm 2.98$ & $0.27 \pm 0.12$ & $0.52 \pm 0.16^{* * \# \#}$ \\
D-dimer (mg/L) & $0.04 \pm 0.01$ & $3.87 \pm 1.24 *$ & $5.25 \pm 1.45^{* *}$ \\
hsCRP (mg/L) & $0.54 \pm 0.18$ & & $7(50)$ \\
Medications & & $20(67)$ & $7(50)$ \\
Beta-blockers, n (\%) & $6(20)$ & $18(60)$ & $9(60)$ \\
Nitrates, n (\%) & $4(13)$ & $20(67)$ & $9(60)$ \\
Statins, n (\%) & $6(20)$ & $12(40)$ & $7(50)$ \\
Calcium blockers, n (\%) & $4(13)$ & & $4.76 \pm 0.24$ \\
ACEI, n (\%) & $7(37)$ & & \\
Left ventricular function & & & \\
EF (\%) & & & \\
LVID (cm) & & & \\
\hline & & & \\
& & & \\
\hline
\end{tabular}

Isolation of DCs

Blood was drawn from an antecubital vein into a vacutainer tube (Beckon Dickinson,USA) containing $3.2 \%$ sodium citrate prior to drug treatment when the patients were admitted to the hospital. Isolation of human peripheral blood mononuclear cells (PBMCs) was performed using Ficoll-Hypaque density gradient centrifugation following the manufacturer's protocol (Human lymphocyte separation medium from Chinese Academy of Medical Sciences Institute of Biomedical Engineering, China).

Purified CD14+ monocytes isolated from PBMCs by MACS were cultured with recombinant human IL-4 (rhIL-4; $100 \mathrm{U} / \mathrm{mL}$, PeproTech, USA) and recombinant human granulocyte-macrophage colony-stimulating factor (Recombinant Human GM-CSF, $100 \mathrm{U} / \mathrm{mL}$, PeproTech, USA) for 7 days. The floating cells with short dendrite were further isolated using CD11c bead-based MACS, which were named peripheral blood DCs with a purity of more than $95 \%$.

\section{RNA interference and transfection}

Small interfering RNAs (siRNAs) were designed and synthesized by RiBoBio Co. Ltd (Guangzhou, China). The sequences of human siRNAs were: KLF2 siRNA: sense 5'-GGA UGA UCU UCG ACG UGG A-3', antisense 5'-UCC ACG UCG AAG AUC AUC C-3; unrelated siRNA: sense 5'-UUC UCC GAA CGU GUC ACG UdTdT-3', antisense 5'-dTdT AAG AGG CUU GCA CAG UGC A-3'. The transfection of siRNA in the cultured human DCs was performed in 6-well plates at 70\% confluence, and at a final concentration of $50 \mathrm{nmol} / \mathrm{L}$ using X-tremeGENE siRNA transfection reagent (Roche). The mRNA level expression was detected after 24 
Fang et al.: Kruppel-Like Factor 2 Regulates Dendritic Cell Activation

hours, while the protein level expression was detected 48 hours later. Evaluation of transfection efficiency: $50 \mathrm{nmol} / \mathrm{L}$ Cy3-labeled unconcerned siRNA was transfected into the cultured VSMCs in 24-well plates as described above. Transfections were performed in triplicate for each treatment. Six hours later, after several washes in PBS, one part was prepared for flow cytometric analyses. The others were mixed in $4 \%$ paraformaldehyde for $30 \mathrm{~min}$ in the dark, washed again with PBS, and cover slips were mounted with PBS/ glycerin. Cells were photographed under a light or fluorescence microscope (for Cy3, wavelength 555nm; Olympus Microscope BX-51).

Preparation of LDL and copper-oxidized LDL

Ox-LDL was prepared as described previously [14].

\section{Cell cultures}

Dendritic cells from health volunteers were prepared as described previously. After dendritic cells transfected with non-trageting siRNA or KLF2 siRNA, cells were stimulated with PBS (control) or $40 \mathrm{ug} /$ $\mathrm{ml}$ ox-LDL for 8 hours. In some experiments, DCs were pretreated for $30 \mathrm{~min}$ with NF-kB specific inhibitor( PDTC, $10 \mu \mathrm{mol} / \mathrm{L}$ ) (Sigma, USA) before further experiments.

\section{Flow cytometric analysis}

The expression of TLR4 and CD80 on DCs was performed by flow cytometry using phycoerythrin (PE) antihuman TLR4 antibody (BioLegeng, USA) and FITC anti-human CD80 (BioLegeng, USA) according to the manufacturer's instructions. Briefly, 20 $\mu$ l PE-conjugated mouse antihuman TLR4 antibody was incubated with $100 \mu \mathrm{l}$ whole blood for $30 \mathrm{~min}$ at room temperature in the dark. The mixture was washed in stain buffer containing bovine serum albumin and sodium azide. Cells were re-suspended in $500 \mu \mathrm{l}$ stain buffer and analyzed by flow cytometer (LSRII, Becton Dickinson, USA). A negative control was prepared by incubating with an isotype-matched control antibody (IgG2a).

\section{RT-PCR analysis}

The level of KLF2 mRNA expression was analyzed by real-time reverse transcription-polymerase chain reaction (Real-time RT-PCR). Cells were harvested from cells or tissues, and total RNA was isolated using Trizol reagent (Invitrogen, USA) according to the manufacturer's recommendations. RNA concentration and its purity were measured by a spectrophotometer. One microgram of total RNA was reversely transcripted into cDNA using RNA PCR Kit (Takara Biotechnology, Japan) and the cDNA was used as a template. The realtime PCR was carried out with Applied Biosystems Step One Real-Time PCR System (Applied Biosystem, USA) according to the manufacturer's protocol. A housekeeping genes GAPDH was used as an internal control for the PCRs. The sequences of the primers were presented as follows: KLF2 sense-primer: 5'-CTA CAC CAA GAG TTC GCA TCT G-3', antisense-primer: 5'-CCG TGT GCT TTC GGT AGT G-3'; GAPDH senseprimer: 5'-TGT TGC CAT CAA TGA CCC CTT-3', antisense-primer: 5'-CTC CAC GAC GTA CTC AGC G-3'. The PCR reaction mixture was prepared with the SYBR Green I (Takara Biotechnology, Japan), cDNA, and the primers following the manufacturer's protocol. The amount of gene expression was then calculated as the difference $(\Delta \mathrm{Ct})$ between the $\mathrm{Ct}$ value of target gene and the $\mathrm{Ct}$ value of GAPDH. Fold changes in target gene mRNA were determined by the formula $2^{-\Delta \Delta C t}$.

\section{Western blot analyses}

Equal protein amounts were loaded onto SDS-PAGE gels. After running gels, proteins were transferred onto nitrocellulose membranes. Membranes were blocked in 5\% nonfat milk and primary antibody incubations were performed with $3 \% \mathrm{BSA}$ (overnight at $4^{\circ} \mathrm{C}$ ). Antibodies used were specific Abs against the KLF2 (Rabbit polyclonal to human KLF2, Abcam, USA). Then membranes were incubated with peroxidaseconjugated secondary antibodies at room temperature for 2 hours. Specific band was detected with chemiluminescence assay (ECL detection reagents, Pierce) and recorded on x-ray film. Designed software was used to quantify the intensities of bands.

Electrophoresis Mobility Shift Assay (EMSA)

Nuclear extracts (15ug per lane) from the DCs were prepared, and mobility-shift analyses were performed as previously described [15]. DNA probes were generated to the NF- $\mathrm{KB}$ consensus sequences 
Fig. 1. The frequency of TLR4 and CD80 positive DCs in normal control, SA and ACS groups. DCs isolated from normal control $(n=30)$, SA $(n=30)$ and ACS $(n=30)$ groups and were sequentially stained with FITC-conjugated anti-CD80 antibody and PEconjugated anti-TLR4 antibody, and analyzed by flow cytometry in gated TLR4 and CD80 positive cells. (A) The TLR4 and CD80 positive cells in normal control, SA and ACS were shown by flow cytometry. (B) Frequencies of TLR4 and CD80 positive cells were analyzed statistically by SPSS 13.0. * indicated vs control, ${ }^{* * *} P<0.001$. \# indicated vs SA group, \#\#\#P<0.001. Data are presented as mean \pm SEM of at least three independent experiments.
$A$
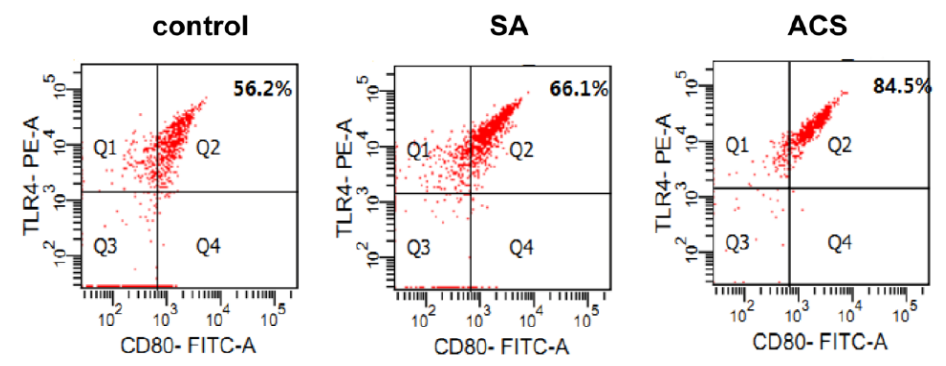

B

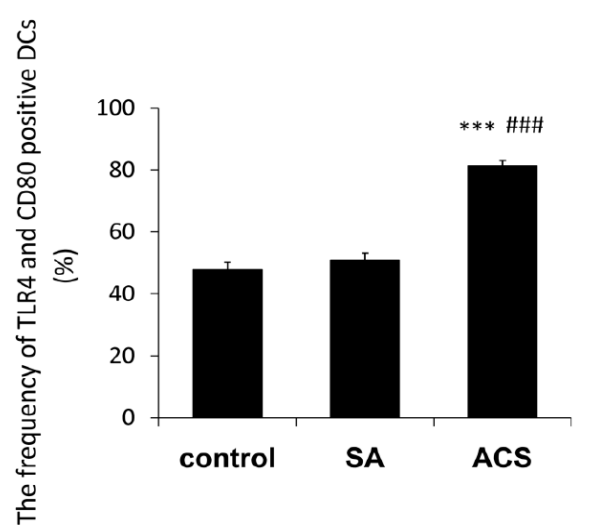

(NF-кB, 5'-AGT TGAGGGGACTTTCCCAGGC-3'). DNA-protein interaction was detected by LightShift TM Chemiluminescent EMSA kit (Pierce). Biotin was labeled at the end of the oligonucleotides. After incubation of nuclear extracts with oligonucleotides at room temperature for $30 \mathrm{~min}$, the DNA binding complexes were run in a $6 \%$ non-SDS polyacrylamide gel for electrophoresis, transferred to nylon membranes (Pierce) and then were cross-linked on a UV transilluminator. Bands were detected with a chemiluminescent method according to the manufacturer's protocol.

\section{Statistical analysis}

Statistics were calculated using ANOVA, by SPSS 13.0. All real-time quantitative RT-PCR determinations were made in duplicate. Measurement data are presented as mean and median \pm SE (mean: median \pm SE). Statistical differences in the amounts of continuous variables between two groups were compared by Wilcoxon test and between more than two groups by the Kruskal-Wallis test. A P-value $<0.05$ was considered significant.

\section{Results}

\section{Clinical Characteristics of Patients}

In patients with ACS and SA, the levels of plasma hsCRP were higher than in patients with normal control $(P<0.01$ and $P<0.05$, respectively). Furthermore, the levels of plasma D-dimer in patients was increased compared with SA and control (all $P<0.01$ ). In addition, there was no significant difference in the levels of plasma D-dimer between normal control and SA patients $(P>0.05)$ (Table 1$)$.

Assessment of the activation of DCs in the different groups

Our study, as well as others, has indicated that TLR4 plays a key role in the activation of DCs $[7,16]$. Furthermore, surface molecules responsible for inducing T-cell activation such 


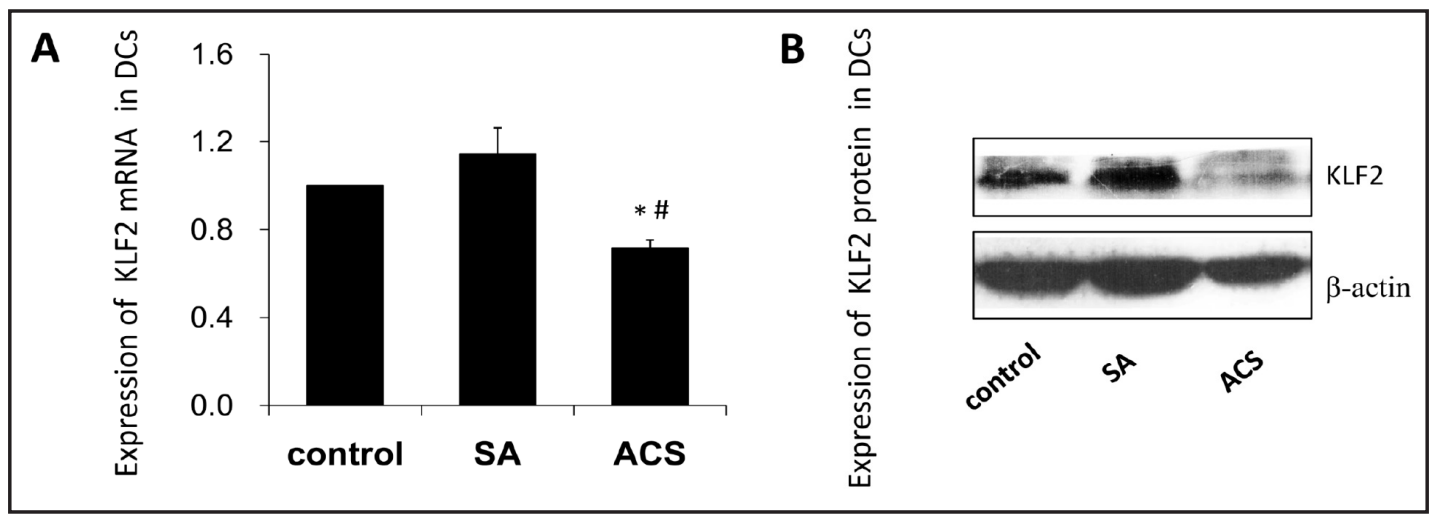

Fig. 2. The expression of KLF2 was decreased in ACS. DCs were isolated from normal control ( $n=30), S A$ $(n=30)$ and ACS $(n=30)$ groups and the expression of KLF2 mRNA and protein in DCs were determined by real-time RT-PCR (A) and Western blot (B). * indicated vs control, ${ }^{*} P<0.05$. \# indicated vs SA group, $\# P<0.05$. Data are presented as mean \pm SEM of at least three independent experiments.

Fig. 3. Ox-LDL decreased KLF2 expression in DCs. DCs were isolated from healthy volunteers and cultured with PBS (control) or ox-LDL (40ug/mL) for 8 hours. After incubation, the expression of KLF2 mRNA and protein in DCs were determined by real-time RTPCR (A) and Western blot (B). (C) DCs were sequentially stained with FITCconjugated anti-CD80 antibody, and the frequency requencies of CD80 positive cells were analyzed statistically by SPSS 13.0. * indicated vs control, ${ }^{* * *} P<0.001$. Data are presented as mean \pm SEM of at least three independent experiments.

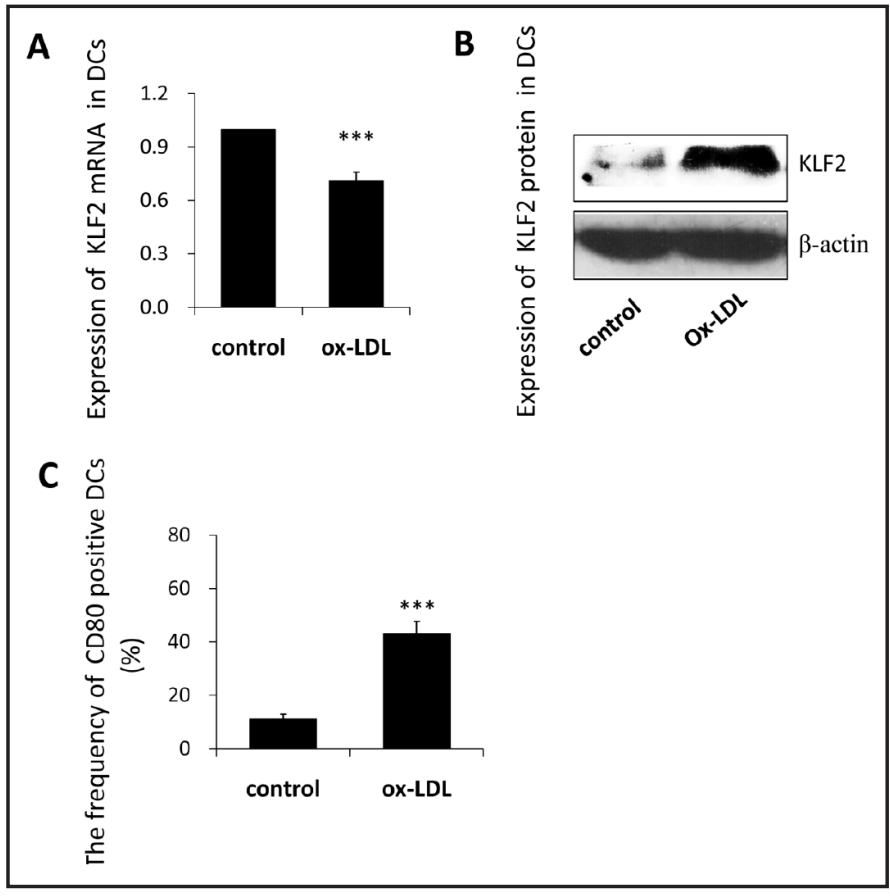

as the co-stimulatory molecules CD80 and CD86, or the major histocompatability complex (MHC) class I and II antigens were involved too [17]. Therefore, the levels of CD80 and TLR4 on surface molecules were used to assess the activation of DCs. Our study found that the surface expression of TLR4 and CD80 for DCs in normal control, SA group and ACS group was $47.75 \% \pm 2.40 \%, 50.9 \% \pm 2.27 \%$, and $81.40 \% \pm 1.64 \%$, respectively. The frequencies of CD80 and TLR4 positive DCs were significantly higher in patients with ACS than in which in SA patients $(P<0.001)$ or normal controls $(P<0.001)$ (Fig. 1$)$. No statistically significant difference was detected between normal control and SA group $(P>0.05)$. Based on these data, we concluded that the state of DCs in patients with ACS was more active than in patients with SA and normal controls.

KLF2 was down-regulated in DCs in the patients with ACS

We next intended to determine KLF2 expression in DCs in normal control, patients with SA and patients with ACS. Real-time PCR showed that compared with normal control; there 


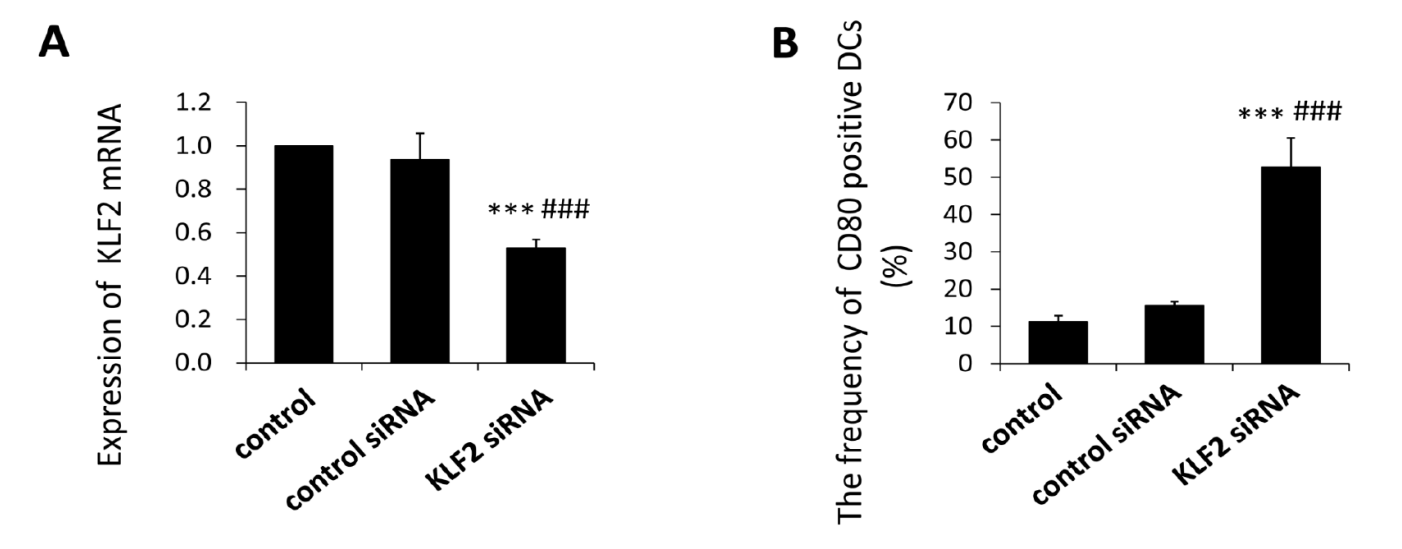

Fig. 4. Down-regulated KLF2 increased CD80 on surface of DCs. DCs were isolated from healthy volunteers and cultured with PBS (control) or transfected with non-targeting siRNA (control siRNA) or siRNA specific for KLF2. (A) After transfection, the expression of KLF2 mRNA and protein in DCs were determined by real-time RT-PCR. (B) DCs were sequentially stained with FITC-conjugated anti-CD80 antibody, and the frequency of CD80 positive cells was analyzed statistically by SPSS 13.0. * indicated vs control, ${ }^{* * *} P<0.001$. \# indicated vs control siRNA, \#\#\#P<0.001. Data are presented as mean \pm SEM of at least three independent experiments.

was no significant change in KLF2 mRNA expression in DCs in patients with SA (normal control: $1.00 \pm 0.00$; SA: $1.14 \pm 0.12)(P>0.05)$. However, there was a marked decrease in KLF2 mRNA expression in DCs in patients with ACS when compared with which in normal control (ACS: $0.72 \pm 0.04$; normal control: $1.00 \pm 0.00)(P<0.05)$ or SA (ACS: $0.72 \pm 0.04$; SA: $1.14 \pm 0.12$ ) $(P<0.05)$ (Fig. 2A). The protein level of KLF2 in DCs was also determined in our study. Coincident with decreased KLF2 mRNA expression, a distinct reduction of KLF2 protein expression was detected in DCs in ACS. In contrast, the difference of KLF2 protein in normal control and SA was not obvious (Fig. 2B).

Decreased KLF2 increased the surface expression of CD80 in DCs

Ox-LDL has been reported to be one of the most important stimuli for DCs activation. Figure $3 \mathrm{~A}$ showed that compared with untreated DCs, ox-LDL stimulation significant reduced KLF2 mRNA expression (control: $1.00 \pm 0.00$; ox-LDL: $0.71 \pm 0.05)(P<0.05)$. Figure 3B showed that level of KLF2 protein was also down-regulated after stimulation with ox-LDL. Furthermore, the surface expression of CD80 was dramatically elevated in DCs stimulated with ox-LDL $(11.20 \% \pm 1.65 \%)$, compared with those cultured with PBS (control: $43.13 \% \pm$ 4.56\%) $(P<0.001)$ (Fig. 3C).

To examine whether there was a causative relationship between the decreased KLF2 and the up-regulated CD80 expession in DCs, we targeted KLF2 in human DCs with siRNA and the expression of CD80 was examined by FACS. Figure 4A showed that compared with non-targeted control siRNA, KLF2 specific siRNA exhibited a strong inhibition on KLF2 mRNA expression $(P<0.001)$ (Fig. 4A). Furthermore, down-regulated KLF2 could modulate DCs activation, as shown by the expression of CD80 significantly increased from $15.58 \% \pm$ $1.10 \%$ on the surface of non-targeted, knockdown DCs to $52.66 \% \pm 7.85 \%(P<0.001)$ (Fig. 4B).

KLF2 -modulated expression of CD80 involved NF- $\kappa B$ pathway

Nuclear factor $\kappa \mathrm{B}(\mathrm{NF}-\kappa \mathrm{B})$, as a crucial transcription factor, is involved in the regulation of inflammatory and immune genes, cell proliferation, and apoptosis. To define whether NF- $\mathrm{B}$ was involved in KLF2-modulated DCs activation, DCs were cultured with siRNA in presence or absence of human NF- $\kappa$ B pathway inhibitor (PDTC, $10 \mu \mathrm{mol} / \mathrm{L}$ ). Then, electrophoretic 
Fig. 5. $N F-\kappa B$ DNA binding activity in DCs. DCs were cultured as described in Figure legend 4. In some experiments, DCs were pretreated for 30 min with NF- $\kappa B$ specific inhibitor (PDTC, 10 $\mu \mathrm{mol} / \mathrm{L}$ ) (Sigma, USA) before further experiments. (A) An electronic mobility shift assay was performed with nuclear exacts (30ug/well) from DCs in different cultures to assay NF- $\kappa B$ activity. (B) DNA binding activity of $\mathrm{NF}-\kappa \mathrm{B}$ was evaluated using the relative method. (C) DCs were sequentially stained with FITC-conjugated anti-CD80 antibody, and the frequency of CD80 positive cells was analyzed statistically by SPSS 13.0. * indicated vs control, ${ }^{* * *} P<0.001$. \# indicated vs control siRNA, $\# \# \# P<0.001$. Data are presented as mean \pm SEM of at least three independent experiments.
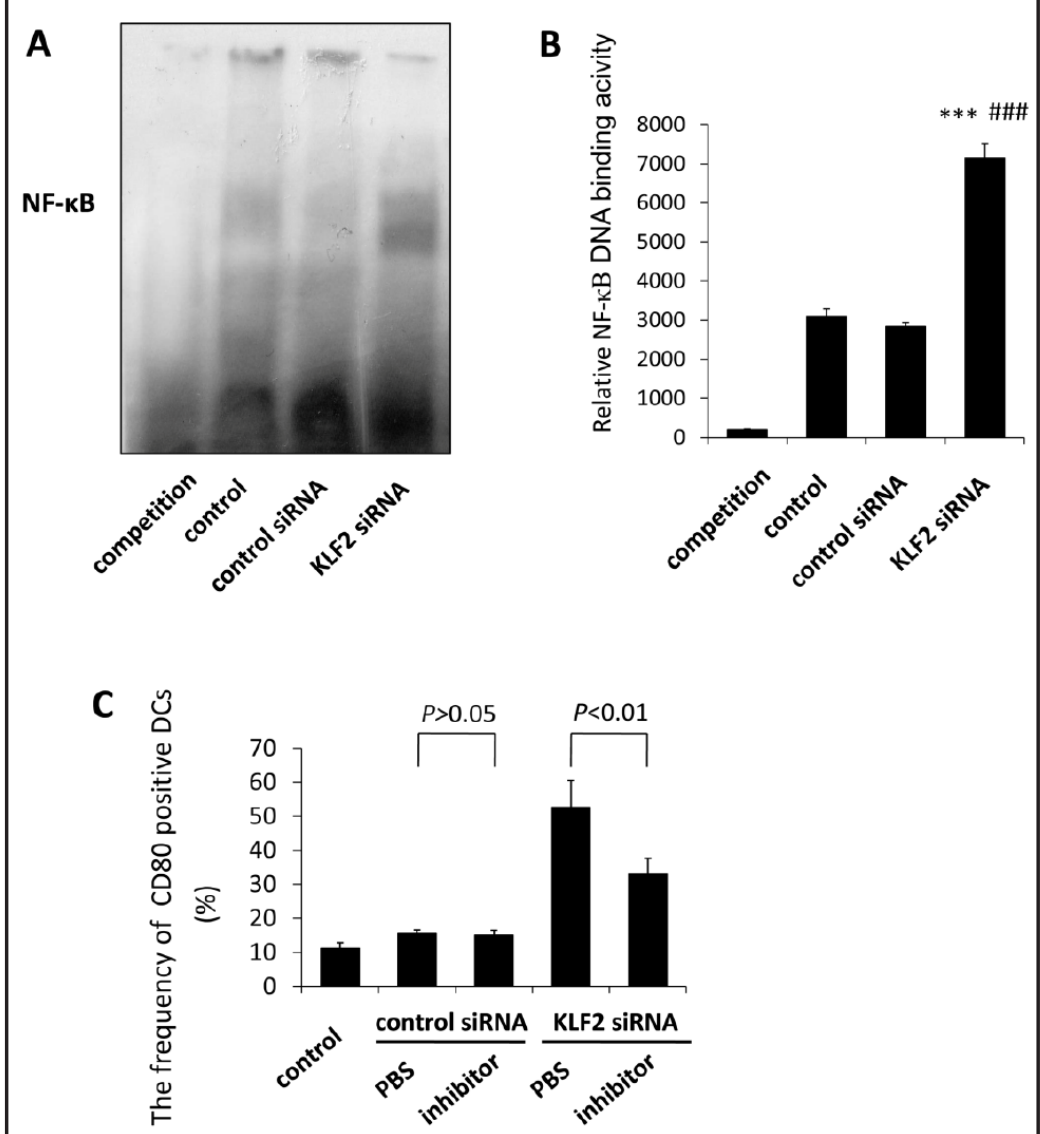

mobility shift assay (EMSA) was performed with specific oligonucleotide probes for the NF$\kappa \mathrm{B}$ binding site regions.

As illustrated in Figure 5A and B, downregulated KLF2 with siRNA resulted in a clear increase in binding of NF- $\mathrm{KB}$ consensus oligonucleotides with DNA (lane 4, integrated option density (IOD) $7152 \pm 358.38$ ), compared with constitutive level of NF- $\kappa B$ activation in DCs without treatment (lane 2, IOD $3095 \pm 204.46)(P<0.001)$ or which transfected with nontargeting siRNA (lane 3, IOD $2840 \pm 100.21$ ) $(P<0.001)$. Furthermore, NF- $\kappa B$ complexes disappeared when competition experiments were performed with an excess of unlabeled NF- $\kappa B$ sequence, demonstrating the specificity of NF- $\kappa B$ DNA-binding (lane 1).

Next we sought to explore whether the increased NF- $\kappa B$ activity in KFL2 knockdown DCs was involved in DCs activation. Figure 5C showed that compared with PBS $(52.66 \% \pm$ $7.82 \%)$, NF- $\kappa B$ specific inhibitor could significantly reduce the surface expression of CD80 in KFL2 knockdown DCs $(33.13 \% \pm 4.57 \%)(P<0.001)$. Therefore, it is plausible to postulate that down-regulated KLF2 modulation leads to CD80 activation via NF- $\mathrm{BB}$ signaling pathway.

\section{Discussion}

Recent research has shown that inflammation plays a key role in coronary artery disease and other manifestations of atherosclerosis. Immune cells dominate early atherosclerotic lesions, their effector molecules accelerate progression of the lesions, and activation of inflammation can elicit acute coronary syndromes [2]. DCs play a key role in 
innate and adaptive immunity [18]. During ACS, circulating DCs are markedly decreased. At the same time, DCs accumulate in vulnerable atherosclerotic tissue [19]. Particularly in vulnerable plaques, previous researches have described a high density of tissue-resident DC with frequent T-cell contacts, suggesting their contribution to plaque destabilization [20]. Dyslipidaemia systemically alters DC function and recent findings suggest that DCs play a role in plaque destabilization [21]. Therefore, DCs play an important role in the origination, development and destabilization of atherosclerosis.

The systemic as well as local vascular immune systems are involved in the development and progression of atherosclerosis. The development and progression of atherosclerosis is mainly determined by endothelial dysfunction with subsequent adhesion, activation and differentiation of leukocytes as well as by the interaction between antigen-presenting cells and T cells [22]. Recently, research has suggested that a reduction of KLF2 expression at levels that approximate those observed in human subjects with coronary artery disease is biologically relevant [23]. The observation provides evidence in support of the hypothesis that KLF2 is atheroprotective in vivo. Previous studies indicate that KLF2 inhibits monocyte proinflammatory gene expression and phagocytosis. Importantly, KLF2 expression in peripheral blood monocytes of patients with established atherosclerotic disease is reduced by $30 \%$ [13]. KLF2 is known to induce eNOS and inhibit VCAM-1 expression in cultured endothelial cells [12]. KLF2 shows reduced expression in response to LPS and in patients with coronary artery disease; its expression is increased by statins. Overexpression represses proinflammatory genes (eg, COX-2, CD40L, MCP-1) [13]. KLF2 is expressed in single-positive $\mathrm{CD}^{+}$and $\mathrm{CD}^{+} \mathrm{T}$ cells, induced by statins and rapamycin, and its expression is rapidly extinguished after T-cell activation via the PI3K/AKT signaling pathway [15, 24-26].

Previous studies about the immune mechanisms of CAD rarely focus on DCs. Research in the regulating effect of KLF2 in activation of DCs, which play a key role in the atherosclerosis, have been rare. In our study, we found that KLF2 probably plays a key role in repressing activation of DCs in patient with ACS.

In the vivo experiment, we showed that the surface expressions of TLR4 and CD80 in the DCs of patients with ACS are much higher than those in the other two groups. The activation of NF- $\kappa B$ in the DCs of patients with ACS is higher than in those of the other two groups. These findings indicate that the DCs of patients with ACS are more activated and mature, and have a higher level of inflammation, than those in the other two groups. We also demonstrated that the levels of KLF2 expression and transcription in the DCs of patients with ACS are lower than those in the other two groups. The levels of KLF2 expression and transcription are negatively correlated with the level of inflammation, activation and maturity. These indices, however, do not distinguish differentiation between group II and group I. Although patients with SA have atherosclerosis in their coronary artery, their lesions are stable and the atherosclerosis is still at a low level of inflammation. We therefore had reasons to believe that KLF2 inhibits DC activation and carried out in vitro experiments to further demonstrate this.

Fragments of bacteria such as lipopolysaccharides (LPs), modified autoantigens such as oxidized LDL, and heat-shock proteins are recognized by TLR4 and activate the subsequent signaling cascade $[27,28]$. Oxidized low density lipoprotein (LDL) is believed to play a key role in the development of atherosclerosis [29]. Therefore, we use oxLDL as the stimulating factor in our in vitro experiment. We found that KLF2 expression and transcription decreased in groups II and IV when stimulated with oxLDL. From the effect of transfection, KLF2 expression and transcription were low or undetected, without obvious diversity. However, the activation of NF- $\kappa B$ of the DCs in group VI was explosively enhanced, which we ascribed to the knockdown of KLF2 gene transcription. Previous studies suggest that NF- $\kappa B$ requires coactivators such as p300CBP or PCAF for transcriptional activation [30, 31]. As such, it is considered that KLF2 may bind to one or more of these coactivators and, as a consequence, render them incapable of assisting NF- $\kappa B$ to induce transcriptional activity. Through the in vivo and in vitro experiments, we have reasoned that Kruppel-like factor 2 (KLF2) signaling inhibits TLR4-induced DC activation in patients with acute coronary syndrome, in which NF- $\mathrm{kB}$ participates. 
Fang et al.: Kruppel-Like Factor 2 Regulates Dendritic Cell Activation

Thus far, the precise signaling mechanisms responsible for activation of DCs in the pathogenesis of atherosclerosis remain ill defined. In this study, we describe for the first time that KLF2 signaling downregulates DC activation in patients with CAD. KLF2 signaling may be a new target of antiatherogenic therapy in the future even though there are challenges to improve understanding of certain areas, such as the actual interaction between TLR4 and KLF2. This underscores the novel therapeutic opportunities that will be gained by modulating TLR4, KLF2 and NF- $\kappa B$ to manipulate innate and adaptive immunity for the immune therapy of CAD.

\section{Acknowledgements}

This work was supported by National Natural Science Foundation of China to Dr. Dazhu Li (No. 30670855 and 81170258 ).

We wish to thank Guiwen Yi, PhD, and Yan Wang, PhD, for their help. They both work in the Department of Cardiology, Institute of Cardiovascular Diseases, Union Hospital, Tongji Medical College, Huazhong University of Science and Technology, Wuhan, China.

All of authors take responsibility for all aspects of the reliability and freedom from bias of the data presented and their discussed interpretation.

\section{References}

1 Ross R: Atherosclerosis is an inflammatory disease. Am Heart J 1999;138:S419-420.

2 Hansson GK: Inflammation, atherosclerosis, and coronary artery disease. N Engl J Med 2005;352:16851695.

3 Banchereau J, Briere F, Caux C, Davoust J, Lebecque S, Liu YJ, Pulendran B, Palucka K: Immunobiology of dendritic cells. Annu Rev Immunol 2000;18:767-811.

-4 Ma-Krupa W, Jeon MS, Spoerl S, Tedder TF, Goronzy JJ, Weyand CM: Activation of arterial wall dendritic cells and breakdown of self-tolerance in giant cell arteritis. J Exp Med 2004;199:173-183.

-5 Pryshchep 0, Ma-Krupa W, Younge BR, Goronzy JJ, Weyand CM: Vessel-specific Toll-like receptor profiles in human medium and large arteries. Circulation 2008;118:1276-1284.

6 Ranjit S, Dazhu L, Qiutang Z, Yibo F, Yushu L, Xiang W, Shen CL, Yuan T: Differentiation of dendritic cells in monocyte cultures isolated from patients with unstable angina. Int J Cardiol 2004;97:551-555.

7 Wang L, Li D, Yang K, Hu Y, Zeng Q: Toll-like receptor-4 and mitogen-activated protein kinase signal system are involved in activation of dendritic cells in patients with acute coronary syndrome. Immunology 2008;125:122-130.

-8 Bieker JJ: Isolation, genomic structure, and expression of human erythroid Kruppel-like factor (EKLF). DNA Cell Biol 1996;15:347-352.

-9 Feinberg MW, Lin Z, Fisch S, Jain MK: An emerging role for Kruppel-like factors in vascular biology. Trends Cardiovasc Med 2004;14:241-246.

-10 Nayak L, Goduni L, Takami Y, Sharma N, Kapil P, Jain MK, Mahabeleshwar GH: Kruppel-like factor 2 is a transcriptional regulator of chronic and acute inflammation. Am J Pathol 2013;182:1696-1704.

-11 Atkins GB, Jain MK: Role of Kruppel-like transcription factors in endothelial biology. Circ Res 2007;100:1686-1695.

-12 Das H, Kumar A, Lin Z, Patino WD, Hwang PM, Feinberg MW, Majumder PK, Jain MK: Kruppel-like factor 2 (KLF2) regulates proinflammatory activation of monocytes. Proc Natl Acad Sci U S A 2006;103:6653-6658.

13 Braunwald E, Antman EM, Beasley JW, Califf RM, Cheitlin MD, Hochman JS, Jones RH, Kereiakes D, Kupersmith J, Levin TN, Pepine CJ, Schaeffer JW, Smith EE, Steward DE, Theroux P, Gibbons RJ, Alpert JS, Eagle KA, Faxon DP, Fuster V, Gardner TJ, Gregoratos G, Russell RO, Smith SC: ACC/AHA guidelines for the management of patients with unstable angina and non-ST-segment elevation myocardial infarction: executive summary and recommendations. A report of the American College of Cardiology/American Heart Association task force on practice guidelines (committee on the management of patients with unstable angina). Circulation 2000;102:1193-1209. 
Fang et al.: Kruppel-Like Factor 2 Regulates Dendritic Cell Activation

14 Lin J, Li M, Wang Z, He S, Ma X, Li D: CD4+CD25+ regulatory T cells in macrophage-derived foam-cell formation. J Lipid Res 2010;51:1208-1217.

15 Schober SL, Kuo CT, Schluns KS, Lefrancois L, Leiden JM, Jameson SC: Expression of the transcription factor lung Kruppel-like factor is regulated by cytokines and correlates with survival of memory T cells in vitro and in vivo. J Immunol 1999;163:3662-3667.

16 Re F, Strominger JL: Toll-like receptor 2 (TLR2) and TLR4 differentially activate human dendritic cells. J Biol Chem 2001;276:37692-3769.

17 Trinchieri G: Interleukin-12 and the regulation of innate resistance and adaptive immunity. Nat Rev Immunol 2003;3:133-146.

18 Nestle FO, Banchereau J, Hart D: Dendritic cells: On the move from bench to bedside. Nat Med 2001;7:761765.

19 Yilmaz A, Weber J, Cicha I, Stumpf C, Klein M, Raithel D, Daniel WG, Garlichs CD: Decrease in circulating myeloid dendritic cell precursors in coronary artery disease. J Am Coll Cardiol 2006;48:70-80.

20 Yilmaz A, Lochno M, Traeg F, Cicha I, Reiss C, Stumpf C, Raaz D, Anger T, Amann K, Probst T, Ludwig J, Daniel WG, Garlichs CD: Emergence of dendritic cells in rupture-prone regions of vulnerable carotid plaques. Atherosclerosis 2004;176:101-110.

21 Bobryshev YV: Dendritic cells in atherosclerosis: current status of the problem and clinical relevance. Eur Heart J 2005;26:1700-1704.

22 Methe H, Weis M: Atherogenesis and inflammation--was Virchow right? Nephrol Dial Transplant 2007;22:1823-1827.

-23 Atkins GB, Wang Y, Mahabeleshwar GH, Shi H, Gao H, Kawanami D, Natesan V, Lin Z, Simon DI, Jain MK: Hemizygous deficiency of Kruppel-like factor 2 augments experimental atherosclerosis. Circ Res 2008;103:690-693.

-24 SenBanerjee S, Lin Z, Atkins GB, Greif DM, Rao RM, Kumar A, Feinberg MW, Chen Z, Simon DI, Luscinskas FW, Michel TM, Gimbrone MA, Garcia-Cardena G, Jain MK: KLF2 Is a novel transcriptional regulator of endothelial proinflammatory activation. J Exp Med 2004;199:1305-1315.

25 McMillan BJ, McMillan SN, Glover E, Bradfield CA: 2,3,7,8-Tetrachlorodibenzo-p-dioxin induces premature activation of the KLF2 regulon during thymocyte development. J Biol Chem 2007;282:12590-12597.

-26 Fabre S, Carrette F, Chen J, Lang V, Semichon M, Denoyelle C, Lazar V, Cagnard N, Dubart-Kupperschmitt A, Mangeney M, Fruman DA, Bismuth G: FOXO1 regulates L-Selectin and a network of human T cell homing molecules downstream of phosphatidylinositol 3-kinase. J Immunol 2008;181:2980-2989.

27 Alderman CJ, Bunyard PR, Chain BM, Foreman JC, Leake DS, Katz DR: Effects of oxidised low density lipoprotein on dendritic cells: a possible immunoregulatory component of the atherogenic microenvironment? Cardiovasc Res 2002;55:806-819.

28 Shen LH, Zhou L, Wang BY, Pu J, Hu LH, Chai DJ, Wang L, Zeng JZ, He B: Oxidized low-density lipoprotein induces differentiation of RAW264.7 murine macrophage cell line into dendritic-like cells. Atherosclerosis 2008;199:257-264.

-29 Shaw PX: Rethinking oxidized low-density lipoprotein, its role in atherogenesis and the immune responses associated with it. Arch Immunol Ther Exp (Warsz) 2004;52:225-239.

-30 Sheppard KA, Rose DW, Haque ZK, Kurokawa R, McInerney E, Westin S, Thanos D, Rosenfeld MG, Glass CK, Collins T: Transcriptional activation by NF-kappaB requires multiple coactivators. Mol Cell Biol 1999;19:6367-6378.

-31 Wardell SE, Boonyaratanakornkit V, Adelman JS, Aronheim A, Edwards DP: Jun dimerization protein 2 functions as a progesterone receptor N-terminal domain coactivator. Mol Cell Biol 2002;22:5451-5466. 